\title{
Using therapeutic groups to support women with faecal incontinence
}

\section{Sonya Chelvanayagam, Julian Stern \\ Abstract}

In this paper, the authors describe the use of facilitated patient groups, in the management of women with faecal incontinence (FI). Two types of groups are discussed - a psychoeducational group and a psychotherapy group. Detailed descriptions of some of the themes which emerged in these groups are provided. The effectiveness of such groups is described, with regard to both psychological and physical functioning. Further investigation into the use of groups for this patient population is recommended.

Introduction

Faecal incontinence (FI) affects $5 \%$ of women (Perry et al, 2002). The most common aetiology is obstetric trauma (Fenner et al, 2003). However there is a wide range of other causes, including neurological diseases, e.g. multiple sclerosis, spina bifida or spinal cord injury (Norton and Chelvanayagam, 2004).

Many women with faecal incontinence are referred to the colorectal service at St Mark's Hospital for biofeedback therapy, for the treatment or management of their symptoms. biofeedback is a wellestablished therapy comprising of a range of interventions which help to both treat and support the individual's symptoms (See Table 1).

Two-thirds of patients receiving biofeedback therapy for faecal incontinence are cured or improved in the short term (Norton and Kamm, 1999). A randomized controlled trial comparing patients who received advice, teaching and information to those who received biofeedback with exercises found no significant differences between the groups (Norton et al, 2003). Patients commented on how therapeutic they found discussing their symptoms with an understanding therapist without fear of rejection (Norton, 2004). Many individuals report feeling stigmatized by their symptoms and are reluctant to disclose their condition (Collings and Norton, 2004).

The purpose of the groups described in this paper, was to assess whether a reliable and safe forum for patients with $\mathrm{FI}$ - where they can discuss some of their personal and interpersonal concerns in a confidential environment - could benefit not only their emotional wellbeing, but also their symptoms of faecal incontinence and global functioning.

\section{Bowels and stigma}

Bowels are a taboo area, even among healthcare professionals (Lawler, 1991). Faeces conjure up feelings of disgust. It is thought this relates to both conditioning as children that stools were 'dirty' and 'smelly', and real fears of contamination from disease (Garcia et al, 2005). In the past, the 
authors have facilitated focus groups to establish the effects of faecal incontinence on quality of life (Chelvanayagam and Norton, 2000). One woman reported that while using a public toilet she heard women comment in a derogatory manner on the smell of faeces. She presumed they were discussing her stools and stated she felt ashamed to leave the toilet and waited some time before leaving the toilet 'and then I scurried away like a little mouse'. As this woman already felt stigmatized by her condition, it is likely she assumed that negative comments surrounding faeces or bowel function could only be attributed to her, even within an area which is deemed appropriate to defecate.

\section{Why group therapy?}

Provision of supportive individual therapy is seen as effective, primarily due to individuals feeling safe enough to disclose their stigmatizing' symptoms to an understanding therapist. However, due to the taboo nature of the symptom and the fears of disclosure, many individuals still feel isolated and disempowered. They feel they are 'the only one' and although they are informed of the prevalence of faecal incontinence within the general population, this does not enable them to see how others in a similar situation manage their incontinence. Ekströmer $(2002, \mathrm{p} 181)$ discuss the issues of body shame and provide a simple and effective example of two children discussing their stomas:

'They were able to talk about how boring it is always to have to spend so much time looking after one's bowel instead of playing. At last! Someone who really understood.'

The authors have facilitated a psychotherapy group for women with faecal incontinence, a psychoeducational group for women with faecal incontinence (Chelvanayagam and Mott, 2005), and a psychotherapy group for women with spinal injuries and bowel disorders (see below).

It has been interesting to observe the similarities and differences between the discussions in both groups. Although they were facilitated using different models of group therapy, much of the discussion focused on the same key issues.

Within a psychoeducational group, the facilitator maintains an active role, setting the format for the group, initiating the discussions, providing support and encouraging each member to contribute. Therefore, there are high levels of facilitator activity. In a psychotherapy group the role of the facilitator is much less directive, and aims to provide an atmosphere to encourage open and honest communication by offering interpretations and feedback to individuals and the whole group (Montgomery, 2002).

\section{Psychoeducational group for women with Fl due to obstetric trauma}

Obstetric trauma usually relates to damage or disruption of the external anal sphincter. The external sphincter is located in the anal canal and is a voluntary muscle which helps to control faecal urgency and maintain continence.

Women with FI who had a poor or limited response to biofeedback therapy were asked if they would be interested in participating in a group. Of the 20 women contacted, 5 were interested and attended an initial group meeting. The women were aged between 37-46 years. All women agreed to attend the groups which occurred monthly for eight months. Each group ran for 90 
minutes and was facilitated by two nurse specialists.

The women selected a range of topics in which they would like to gain knowledge and information: neurological functioning of the bowel, urinary incontinence, psychological aspects, and general discussion regarding managing $\mathrm{Fl}$, such as bowel irrigation. The format of the group allowed for discussion on these areas and also provided a supportive forum for the women.

The main themes discussed throughout the groups were disclosure of symptoms, seeking help and treatment, availability and accessing toilets, hidden disability, and psychological aspects (see Table 2).These themes are reported in greater depth elsewhere in the literature (Chelvanayagam and Mott, 2005).

To assess the outcome of this group the women completed the Manchester Health Questionnaire (Bugg et al, 2001) and the Hospital and Anxiety Depression (HAD) Scale (Zigmond and Snaith, 1983) before starting the group sessions and after the final group meeting.

The Manchester Health Questionnaire is a self-report quality of life measure developed specifically for women with $\mathrm{FI}$ which could also be used as an evaluation tool for postpartum women. The HAD Scale is a validated screening tool for measuring anxiety and depression for patients with physical symptoms. After the final group meeting they also completed a specifically developed evaluation form.

\section{Evaluation of the group}

Prior to the start of the group meetings, the women reported that their symptoms of $\mathrm{Fl}$ had caused a detrimental effect on their quality of life and these effects were generally unchanged after the group sessions. However, there was a noticeable improvement in domain of social functioning. Questions in this domain relate to limitations in social life, visiting friends and the effects on family life. Therefore, it was felt that participation and support within these groups may have provided increased confidence to the women to allow for socialization in other areas.

The HAD Scale did not identify significant levels of anxiety and/or depression prior to or after the group meetings. Overall comments on the evaluation form stated that, although there had been no change in their physical symptoms, psychologically they felt much better with increased confidence:

'It has had a great positive psychological effect. It has been good to realize that there are other people suffering from the same problem and this has made it easier to accept my own condition.'

They enjoyed and appreciated the group meetings:

'I think the format of the group was great, it felt fluid not rigid which enabled people to relax more. A support network is vital and this group provided me with one.'

\section{Psychotherapy group for women with spinal injuries and bowel dysfunction}

Clinicians involved in the care of individuals with spinal injury and associated bowel disorder have reported that these patients- many of whom suffer from isolation and stigmatization - may benefit from group therapy.

The participants in the psychotherapy group - all women - were recruited from the population of female patients attending the hospital who had a diagnosis of cauda equina syndrome (Figure 1) or spina bifida (Figure 2), and associated bowel dysfunction or Fl. 
The authors arranged to see each patient individually for an initial appointment. Of the 16 suitable patients, eight agreed to attend an initial appointment, but only five attended. Of these five, two women decided after initially agreeing to the group that they would be unable to attend due to recurrent illness and travel difficulties. Therefore this group consisted of three women aged between 30-38 years. Two women had developed cauda equina syndrome and one woman had been diagnosed with spina bifida.

Cauda equina syndrome is recognized as an acute surgical emergency. It results from the nerve roots of the lower lumbar spine becoming compressed (usually by a prolapsed disc), with a rapid development of neurological impairment. The main features are acute low back pain, urinary and/ or bowel dysfunction, weakness in both legs and loss of sensation or abnormal sensation in perineal area and over the buttocks (also known as saddle anaesthesia). If urgent neurosurgical decompression does not occur, the person may retain any or all of these symptoms (Markham, 2004). The two women in the group had lost or altered sensation in buttocks, perineal area, inner thighs, back pain, difficulty walking and had urinary and faecal bowel dysfunction.

Spina bifida literally means 'split spine' (Association for Spina Bifida and Hydrocephalus (ASBAH), 2003) It is a congenital condition involving incomplete development of the spinal column.

All three women in the group reported bladder and bowel dysfunction, back pain, loss of sensation or abnormal sensation of perineal area and buttocks and difficulty walking. The two women diagnosed with cauda equina syndrome had a sudden onset of disability, whereas the woman with spina bifida had lived with increasing disability throughout her life.

The women were sent questionnaires prior to the start of the group and after the groups terminated. These were Short Form-36 (SF36) and the HAD Scale. The SF36 (Ware and Sherbourne, 1992) is a widely used generic health-related measure which examines quality of life in nine domains:

- Physical functioning

- Physical roles

- Pain

- General health

- Vitality

- Social functioning

- Emotional roles

- Mental health

- Health transition.

The results from the SF36 questionnaire reported an adversely affected quality of life in all the domains. The responses from the HAD questionnaire were similar to those in the psychoeducational group in that there were no significant levels of anxiety or depression reported.

The group was facilitated by a consultant psychotherapist and a nurse specialist. Nine group sessions were held, the first seven occurred weekly and the last two sessions were held following a month break. The duration of each group was 90 minutes. Initially, the women reported feeling uncomfortable as the facilitators did not dictate a formal structure. They requested that the facilitators suggest a topic to enable discussion. Although this formal structure was never created, this did not deter the women from discussing a range of topics which became the main recurring themes for the duration of the group meetings (see Table 3 ). 


\section{Symptom checking and disclosure of symptoms}

At the start of the first group meeting onewoman began by asking the other participants their diagnosis and symptoms. In a similar fashion to the psychoeducational group members, they reported how therapeutic it felt to be able to discuss their symptoms freely without fear of being stigmatized. They reported feelings of isolation, with a reluctance to disclose either their bowel symptoms or their level of physical disabilities even to those closest to them.

The women with cauda equina syndrome (due to the rarity of the condition) had never met others with the same condition and were pleased to be able to discuss with each other the level of their disability. They compared their quality of life before and after their injury, while the participant with spina bifida reported a gradual worsening of symptoms over her life span, and no experience of life without a disability.

\section{Experiences of health services}

Similar to the women in the psychoeducational group discussed earlier, all the women reported negative experiences of health services and healthcare professionals. They reported situations where they had been ignored or humiliated, and a worsening of symptoms had occurred leading to further medical interventions. Although they also reported positive experiences with healthcare professionals, these were greatly overshadowed by the frequency and severity of their negative experiences.

As the facilitators were also healthcare professionals, we questioned their relationship with us. They reported feeling in control of this situation, possibly because they were aware that the format of the group was prescribed by them and not the facilitators. Benson (2001) reports that there is a period within the time span of group meetings called the 'control stage'. It represents a period of transition from 'less intimate to more intimate system of relationships within the group' (Benson, 2001 p103). However, it is called the 'control stage' refers to issues relating to the competition for leadership. This was certainly demonstrated by the women within the group meetings, and outside the group meetings. They started meeting (informally, in the waiting area) each week before the official start time of the group which invariably meant they were late for the start of the formal group. This is an example of the members of the group appropriating control, and perhaps giving the facilitators a taste of the impotence they (the group members) feel on a daily basis.

\section{Litigation}

The women's negative experiences had led all three women to consider litigation. In essence, they stated that an acknowledgment and an apology by the relevant healthcare professional would alleviate their distress and litigation would not be considered. Anthony (2005) confirms that this can sometimes be the case. He reports that the evidence from studies shows that if patients are informed that an error has occurred, an apology is offered, along with the reassurance that action is being taken to prevent a similar error occurring, they are less likely to pursue litigation.

The consensus of opinion from the women was that even a successful outcome of litigation would not provide them with what they most desired - an improvement in their health or a return to their level of functioning before the injury occurred.

\section{Loss and quality of life}

Discussions surrounding loss were related to the loss of a life that they may have lived or had lived and lost. When discussing everyday activities that many take for granted, each woman had her own 
strategy to complete these activities. These activities required planning and preparation and, to some extent, a reliance on friends, family and partners which they found difficult to accept. They compared their current quality of life to their situation prior to their injury or worsening of symptoms and at times became visibly distressed.

There was a range of emotional responses in the group members, with one group member somewhat stuck in a state of resentment and feeling that very little improvement was possible, while the other two women seemed more able to grieve and move on.

\section{Sexual functioning}

The issue of sexual functioning was raised frequently, sometimes obliquely and sometimes more explicitly. The two women with cauda equina syndrome reported a loss of perineal sensation which had a direct effect on sexual functioning and pleasure. However, for the woman with spina bifida, sensation in the genital area had been absent since birth. She stated, 'as I've never felt anything I don't feel as if I've lost anything'.

One of the women with cauda equina reported pain during coitus which had led her to avoiding sexual contact with her partner. The other patient with cauda equina had avoided entering into any new sexual relationship since the acute onset of her condition.

This was clearly an area they wished to discuss further but was always raised towards the end of the group meetings. It also became evident that this was an area discussed by the group members outside the official group meetings.

The facilitators speculated whether this was due at least or in part, to the psychotherapist being a man. However, when this was discussed, the women stated this did not inhibit their discussion. (In other groups that the authors have facilitated, women have reported that the presence of men would inhibit discussion around sexual issues (Chelvanayagam and Norton, 2000).

It is likely that there were a number of reasons for the discussion taking place partly inside and partly outside the sessions, including: embarrassment; taking of control by the participants; and an 'acting out', whereby the group members gave the facilitators a sense of what it is like to be excluded or kept in the dark (Stern, 2005).

\section{Disability and hidden disability}

All participants reported having not only a physical disability but also a hidden disability caused by their spinal injury, such as their bowel and bladder dysfunction. They found that peoples' responses to their physical disability varied. As many of their symptoms of disability were hidden (e.g. bowel and bladder dysfunction, loss of sensation and limb weakness), they expressed that they were perceived 'not to be disabled enough'. This was particularly relevant when they applied for financial assistance or services.

\section{Employment}

The women's disabilities had also affected their employment situation; either being informed that they were not suitable to work in their departments, or through concern about the alterations required by the employer to be in line with the Disability Discrimination Act 1995. One of the women was attending a job interview and was unsure how much to disclose. There was a consensus between the women that the disclosure should consist of what was necessary and not about bowel and bladder dysfunction. Joachim and Acorn (2000) state that a person with a chronic condition may suffer from both visible and invisible stigma. A visible stigma is one that is 
immediately apparent to the observer, such as a person with unusual gait, and therefore it is difficult to conceal their disability. However, a bowel dysfunction is not immediately apparent and therefore can be concealed so is perceived as an invisible stigma. Therefore, as this woman discovered, although she did not have a choice regarding her physical disability, she could choose whether to disclose her bowel and bladder dysfunction.

\section{Evaluation of the group}

Two of the three participants reported substantial improvements in both their physical and mental health, with an equivocal outcome for the third. In the last group, one woman with cauda equina syndrome reported improved functioning and sensation in her limbs and had successfully returned to full-time work. Both women with cauda equina syndrome completed the outcome measures, and for both, there was a noticeable improvement in their social functioning score on the SF36 quality of life scale (which also occurred for the women in the psychoeducational group). They reported that what they found most helpful about the groups was 'talking to others in a similar situation'. They reported that they did not like the lack of structure. However, despite or perhaps because of the lack of structure, the women expressed and explored primitive and complicated anxieties which a tighter structure may have inhibited.

\section{Conclusion}

This article has discussed two different types of therapeutic groups with women who experience bowel dysfunction. Despite differences in group therapy style the women appreciated the opportunity for discussion in a supportive confidential setting. Although it could have been predicted that psychological improvements would be made, an unexpected finding was the definite physical improvement and improved quality of life in two of the three women in the psychotherapy group.

The authors recommend that further investigation of the use of groups for the support and treatment of individuals with bowel dysfunction and disorders is required. These groups have provided these patients with a therapeutic forum to discuss their fears, hopes and expectations with others in a similar situation. The confidential and safe setting, the sense of catharsis, of cohesiveness and interpersonal learning were some of the therapeutic factors for these patients. These factors, allied with careful empathetic support from the group facilitators and from the other patients in both groups,facilitated improvements in both the physical wellbeing, and the psychological functioning of patients with faecal incontinence

\section{References}

Anthony S (2005) Forgiving fallibility. Casebook Journal of Medical Protection Society 13(3): 8-12

Association for Spinia Bifida and Hydrocephalus (2003) What is Spinia Bifida?: Information Sheet 1. Available: www.asbah.org/UserFiles/InfSheet_1-SB.pdf. Accessed 29 September 2006

Benson JF (2001) Working More Creatively With Groups 2nd edn. Routledge, London

Bug GJ, Kiff ES, Hosker G (2001) A new condition- specific health-related quality of life questionnaire for the assessment of women with anal incontinence. BJOG 108: 1057-67

Chelvanayagam S, Mott L (2005) Supporting women with faecal incontinence. Gastrointestinal Nursing 3(10): 28-32

Chelvanayagam S, Norton C (2000) Quality of life with faecal continence problems. Nurs Times 96(31 suppl):15-7 
Collings S, Norton C (2004) Women's experiences of faecal incontinence: a study. Br J Community Nurs 9(12): 520-3

Ekströmer A (2002) Body Shame In Children Who Have Bowel Disorders. In: Gilbert P, Miles J eds. Body Shame: Conceptualisation, Research and Treatment. Brunner Routledge, Hove: 171-85

Fenner DE, Genberg B, Brahma P, Marek L, DeLancey JO (2003) Fecal and urinary incontinence after vaginal delivery with anal sphincter disruption in an obstetrics unit in the United States. Am J Obstet Gynecol 189(6): 1543-9

Garcia JA, Crocker J, Wyman JF, Krissovich M (2005) Breaking the cycle of stigmatisation. J Wound Ostomy Continence Nurs 32(1): 38-52

Joachim G, Acorn S (2000) Stigma of visible and invisible chronic conditions. J Adv Nurs 32(1): 243-8

Lawler J (1991) Behind the Screens: Nursing, Somology and the Problem of the Body. Churchill Livingstone, Melbourne

Markham DE (2004) Cauda equina syndrome: diagnosis, delay, and litigation risk. Journal of Medical Defence Union 20(1): 12-5

Montgomery C (2002) Role of dynamic group therapy in psychiatry. Advances in Psychiatric Treatment 8: 34-41

Norton C (2004) Nurses, bowel continence, stigma and taboos. J Wound Ostomy Continence Nurs 27: 279-91

Norton C, Chelvanayagam S (2001) Methodology of biofeedback treatment for adults with faecal incontinence. J Wound Ostomy Continence Nurs 28(3): 156-68

Norton C, Chelvanayagam S (2004) Bowel Continence Nursing. Beaconsfield Publishers, Beaconsfield

Norton C, Chelvanayagam S, Wilson-Barnett J, Redfern S, Kamm M (2003) Randomised controlled trial of biofeedback for fecal incontinence Gastroenterology 125(5): 1320-9

Norton C, Kamm MA (1999) Outcome of biofeedback for faecal incontinence. Br J Surg 86: 1159-63

Perry S, Shaw C, McGrother C et al (2002) The prevalence of faecal incontinence in adults aged 40 years or more living in the community. Gut 50: 480-4

Stern J (2005) Psychotherapy: Individual, Family and Group. In: Wright P, Stern J, Phelan M eds. Core Psychiatry 2nd edn. Elsevier Saunders, London

Ware JE Jr, Sherbourne CD (1992) The MOS 36-item short form health survey (SF36). I. Conceptual framework and item selection. Med Care 30: 473-83

Zigmond AS, Snaith RP (1983) The hospital anxiety and depression scale. Acta Psychiatr Scand 67: $361-70$

Table 1. Elements of Biofeedback therapy

Diary and symptom questionnaire

Assessment and patient teaching

Biofeedback: sphincter exercises \&.balloon distension

Urge resistance programme

Management of faecal incontinence

Lifestyle modifications

Support and encouragement

Access to multidisciplinary team

Source: Norton and Chelvanayagam (2001) 
Table 2. Main themes from psychoeducational group meetings

Disclosure of bowel symptoms

Seeking help and treatment

Availability and accessing toilets

Hidden disability

Psychological aspects

Table 3. Main themes from psychotherapy group meetings

Symptom checking

Disclosure of bowel and physical symptoms

Experiences of health services

Litigation

Loss

Sexual functioning

Disability \& hidden disability

Employment

\section{KEY POINTS}

Faecal incontinence affects $5 \%$ of women and can have a detrimental effect on their physical, psychological and social functioning.

As bowel function is a taboo area, many women feel stigmatized, isolated and are reluctant to disclose their symptoms.

Treatment of faecal incontinence with an understanding nurse specialist was reported to be therapeutic (Norton, 2004), however many women still feel isolated.

Group therapy, facilitated by experienced therapists provides a forum for women to discuss their symptoms and ef fects on their quality of life with other women experiencing faecal incontinence. This can lead to an improvement in both the physical and psychological wellbeing

Further investigation of group therapy is recommended to support and treat patients with bowel dysfunction. 


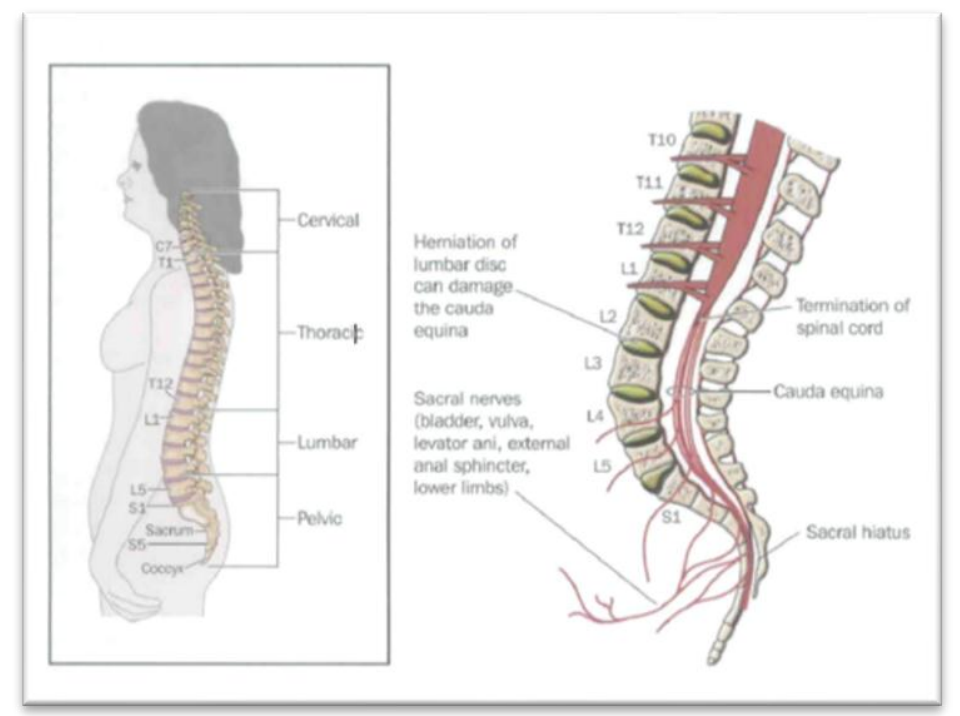

Figure 1. Cauda equine syndrome

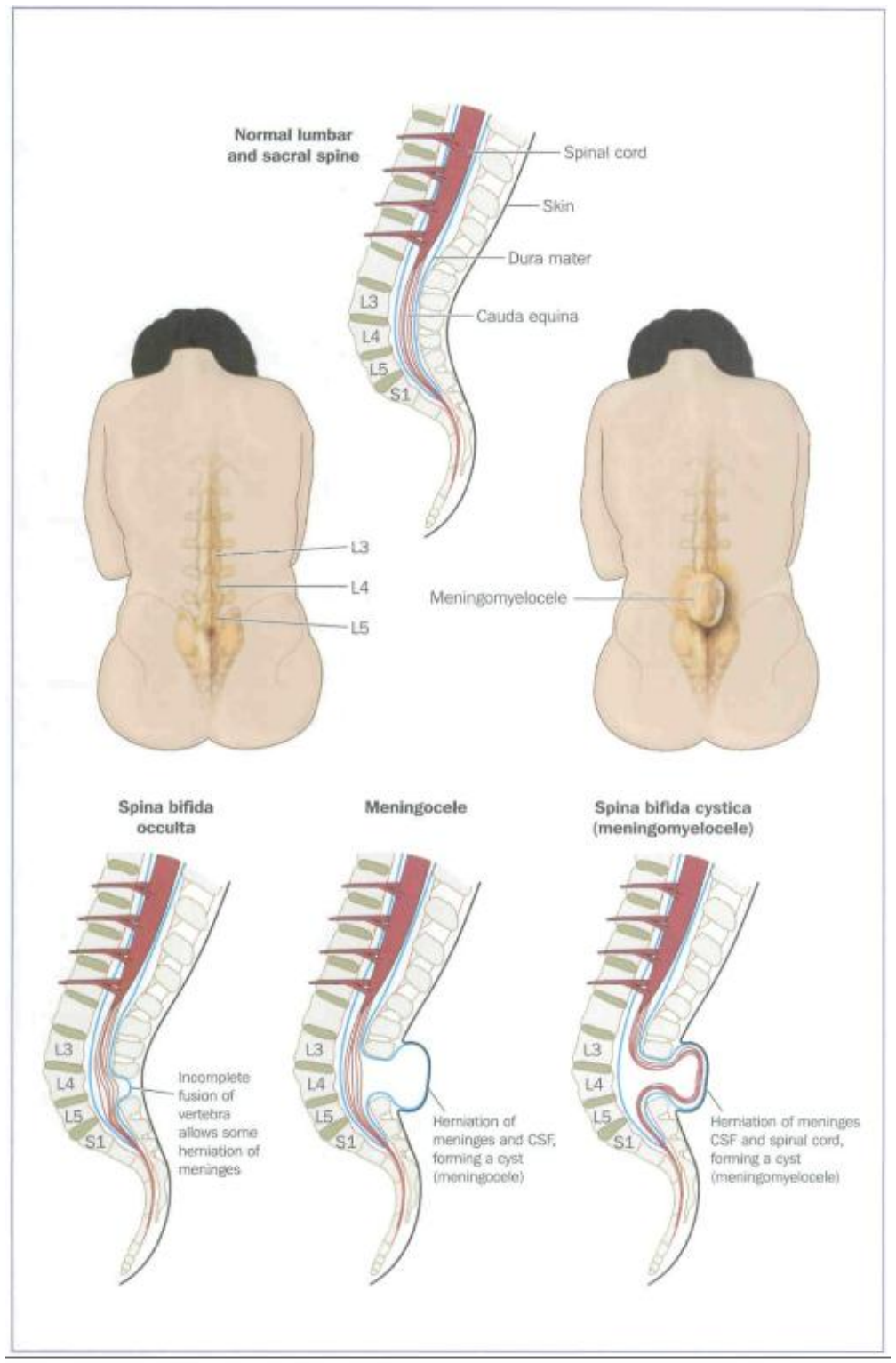

Figure 2. Spina binfida 